\title{
Suicide Ideation in Older Adults Recovering from Acute Conditions in a Clinical Recovery Facility
}

\section{ABSTRACT}

The aim of the current study was to assess suicide ideation in a sample of older adults recovering from acute conditions in a clinical recovery facility of the Portuguese Red Cross. Four indicators of suicide ideation were used. Clinical, contextual, and psychological variables-namely, previous suicide attempts, death of a family member by suicide, loneliness, and interpersonal needs-were also tested to determine whether they were related to suicide ideation. Findings show suicide ideation is frequent at admission and related to patients' previous suicide attempts. Death of a family member by suicide and patients' functional dependency are related to some indicators of suicide ideation, and loneliness and interpersonal needs are important psychological variables relating to suicide ideation. Interpersonal needs were related to suicide ideation even after controlling for previous suicide attempts. Implications of the results for clinical practice are discussed. [Journal of Gerontological Nursing, 43(9), 31-37.]

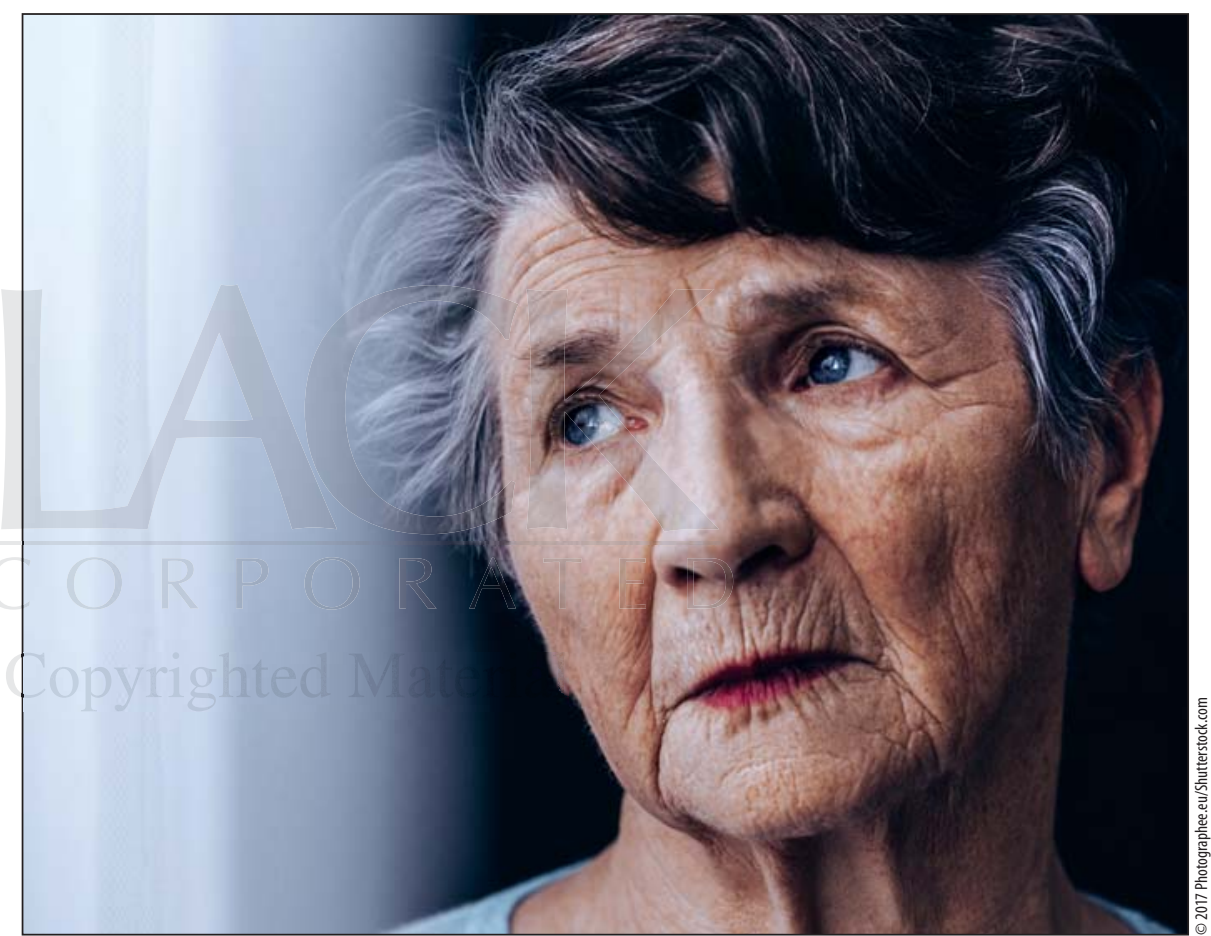

S uicide is an important public health problem. Every year, more than 800,000 individuals die by suicide (World Health Organization, 2017), and it has high incidence rates in older adults (Cukrowicz, Jahn, Graham, Poindexter, \& Williams, 2013; Overholser, Braden, \& Dieter, 2012). Moreover, death rate by suicide increases in the population of those older than 65 (with a ratio of four attempts to one suicide versus 25 attempts to one suicide for all ages). In individuals older than 75 , the death rate can reach two or three times that of cohorts of individuals 25 and younger in most industrialized countries (Cukrowicz et al., 2013).

In Portugal, suicide rates remained stable in the period between 2007 and 2013-at approximately 10 per

Rui C. Campos, PhD; Sara Santos, PhD; Margarida Piteira, MA; Marta Abreu, MA; and Sofia Tavares, PhD 